\title{
Democracias fragmentadas, control del poder y principio de responsabilidad. Un nuevo constitucionalismo en la era del mercado global*
}

\author{
Jordi Jaria i Manzano \\ Profesor agregado interino de Derecho constitucional \\ Universitat Rovira i Virgili
}

Recibido: 01.10 .2012

Aceptado: 15.10 .2012

Resumen: El poder político está experimentando una reorganización en los últimos decenios, de manera paralela al proceso de integración global de los mercados. El estado-nación se ha visto superado en este nuevo marco, lo que plantea nuevas exigencias al constitucionalismo a la hora de responder a los retos tradicionales que se había planteado en relación con la limitación del poder y la garantía del status de los ciudadanos. Ante ello, es necesario pensar en un marco constitucional global, basado en la fragmentación, el pluralismo y la responsabilidad.

Palabras clave: democracia, control del poder, principio de responsabilidad.

Abstract: Political power is experiencing a deep reorganisation in last decades, parallel to process of global integration of markets. Nation-state is somehow superseded within this new scenario. That implies new challenges to constitutionalism in order to design updated tools to guarantee the limitation of power and the status of citizens. For this reason it is required to think about a new global constitutional framework, based in fragmentation, pluralism and responsibility.

Key words: democracy, control of the public power, principle of responsibility.

Sumario: 1. Introducción: la fase global del proceso de acumulación capitalista.-2. El (¿nuevo?) papel del estado-nación como estructura institucional del proceso de acumulación capitalista.-3. Tecnología, velocidad y fragmentación. Una nueva división de poderes-4. De democracias fragmentadas e instrumentos de control global.-5. Rompiendo con el progreso: adaptación al medio y responsabilidad en un nuevo constitucionalismo.-6. Conclusión.

* Esta comunicación forma parte del trabajo del autor en el marco del proyecto de investigación Estado autonómico y democracia: los derechos de participación en los Estatutos de Autonomía, financiado por el Ministerio de Educación y Ciencia para el período 2009-2012 (DER2009-12921/ subprograma JURI), y coordinado por el Prof. Josep M. Castellà Andreu. 


\section{INTRODUCCIÓN: LA FASE GLOBAL DEL PROCESO DE ACUMU- LACIÓN CAPITALISTA}

No puede desconocerse la vinculación entre la aparición del estado moderno y la construcción de los respectivos mercados interiores. Como ya he defendido en otra parte, el estado supone la estructura institucional de la civilización moderna, que se despliega en el proceso de acumulación capitalista -que constituye su forma de metabolismo social-, con la tecnociencia como forma de organización de la experiencia y de estar en el mundo, y que se construye alrededor de la idea cartesiana de sujeto abstracto y racional ${ }^{1}$. Efectivamente, en este contexto, el estado se presenta como condición de posibilidad institucional para el despliegue del proceso de acumulación capitalista, garantizando un espacio normativo homogéneo y seguro -articulado entorno a la idea de seguridad jurídica-, así como la movilidad de las personas y los recursos ${ }^{2}$.

Debe notarse que el mercado, como espacio homogéneo e integrado en el que se produce el proceso de acumulación capitalista, tiende hacia la uni$\mathrm{dad}^{3}$. Sin embargo, en las fases previas del proceso de acumulación capitalista,

${ }^{1}$ Vid. JaRia I Manzano, J., La cuestión ambiental y la transformación de lo público, Tirant lo Blanch, Valencia, 2011, pp. 17 ss.

${ }^{2}$ El estado de Derecho, en sus dimensiones formal y material, supone, justamente, el aseguramiento de ambas dimensiones. La importancia de la predicibilidad del Derecho en la construcción de la propia idea de estado de Derecho en sentido formal es subrayada, por ejemplo, por HäFELIN, U., y HALLER, W., en Schweizerisches Bundesstaatsrecht, Schultess, Zúrich, 2001, p. 52. En cuanto a la idea de los derechos como esfera para el despliegue del proyecto de vida propio de los individuos en un marco abierto de relaciones sociales - por oposición a la sociedad estamental general-, se corresponde con la propia idea de sujeto, central en la comprensión del mundo propia de la modernidad europea, que se proyecta en el contractualismo como fundamento del poder político. El contractualismo aparece ya en los primeros tiempos de la Edad moderna -así, por ejemplo Althusius (vid. STOLLEIS, M., Histoire du droit public en Allemagne. Droit public impérial et science de la police 1600-1800, PUF, París, 1998, pp. 154 ss.-, aunque encuentra su formulación clásica en Hobbes (vid., en particular, HobBES, T., Leviatán, Alianza, Madrid, 1989, p. 144) y Locke (vid. LockE, J., Segundo Tratado sobre el gobierno civil, Alianza, Madrid, 1990, pp. 36 ss.).

${ }^{3}$ Es cierto que ha habido modos alternativos al mercado en el despliegue del proceso de acumulación capitalista, verbigracia el caso de la planificación centralizada de tipo soviético. Debe notarse, en primer lugar que, efectivamente, se trata de un modelo de acumulación capitalista. Hablaríamos, en este caso, de capitalismo de estado, donde este dirige la transformación de la naturaleza en capital, con la diferencia de que los resultados de dicha operación no se distribuyen a través del mercado, sino que el reparto se efectúa de modo dirigido y planificado. Se podría hablar, en este sentido, «acumulación socialista de capital», como lo hace Wallerstein, I., en Geopolítica y geocultura. Ensayos sobre el moderno sistema mundial, Kairós, Barcelona, 2007, p. 127. En el mismo sentido, subrayando su impacto sobre los recursos naturales, vid. GoRz, A., «Ecología y libertad», en Crítica de la razón productivista, Libros de la Catarata, Madrid, 2008, p. 73; y MARTínez Alier, J., «Conflictos ecológicos y justicia ambiental», Papeles de relaciones ecosociales y cambio global n. 103, 2008, p. 13. En segundo lugar, lo cierto es que es un modelo históricamente superado a partir de la Caída 
no nos hallamos ante un espacio global plenamente integrado, sino ante la existencia de «mercados nacionales», por una combinación de razones de tipo geopolítico y, sobre todo, tecnológico. Dichos mercados nacionales, a partir del siglo XIX, devienen «mercados imperiales», con la expansión de los estados europeos en todo el mundo, que se integrarán en un mercado global en la compleja fase histórica que se despliega a partir del final de la Segunda Guerra Mundial, en que la hegemonía económica mundial pasa, definitivamente, a los Estados Unidos ${ }^{4}$.

En cualquier caso, debe notarse que el estado y el mercado son realidades paralelas que aparecen en Europa al final de la Edad media y que se despliegan a nivel global en el marco de lo que ha dado en llamar el sistema-mundo capitalista. En este contexto, se produce una separación de lo público (el poder) y lo privado (los derechos), que constituye la matriz del constitucionalismo como tradición política emergente en el marco de dicho proceso histórico ${ }^{5}$. Sin embargo, a partir de que las posibilidades tecnológicas - nuevas tecnologías de la información y la comunicación- y geopolíticas -constelación imperial de estados bajo hegemonía norteamericana, que se consolida entre el final de la Segunda Guerra Mundial y el fin de la Guerra Fría-, el mercado y el estado dejan de estar en sintonía, generándose estructuras de poder más allá del estado basadas en las posibilidades abiertas por la nueva fase del proceso de acumulación capitalista ${ }^{6}$.

Así, a partir del impulso generado por las nuevas tecnologías de la información y la comunicación (velocidad) y la inercia del proceso de acumulación capitalista (cantidad), los mercados tienden hacia la integración global, mientras que el espacio político teórico continúa determinado por el estado-

del Muro, en 1989, cuando se inicia la fase final del período de integración global de los mercados, una vez derrotado el socialismo real.

${ }^{4}$ Para una visión panorámica de este proceso, es de consulta obligada desde su aparición el trabajo magno de Fontana, J., Por el bien del imperio. Una historia del mundo desde 1945, Pasado\&Presente, Barcelona, 2011.

${ }^{5}$ Así, a partir de la separación de los individuos - presupuesto de la comunidad política- y la estructura de poder, que se basa en una concepción individualista y abstracta del ser humano - «the most important artefact of European history, fundamental for self-understanding of most individuals in the Western world», según VON BOGDANDY, A., "Constitutional Principles for Europe», en Riedel, E., y Wolfrum, R. (Eds.), Recent Trends in German and European Constitutional Law, Springer, Berlín-Heidelberg-Nueva York, 2006, p. 7-, se produce la separación entre lo público y lo privado, en la que el mercado y el estado se reparten los papeles en el despliegue de la vida social.

${ }^{6}$ Así, las transacciones y las conexiones organizativas que se despliegan más allá de las fronteras de los estados van cobrando importancia, determinando la generación de nuevas estructuras de poder en red de alcance global y definiendo el proceso que se ha dado en llamar 'globalización'. Vid., en este sentido, Evans, P., «¿El eclipse del Estado? Reflexiones sobre la estatalidad en la época de la globalización», en CARBONELL, M., y VÁZQUEZ, R. (Eds.), Globalización y Derecho, Ministerio de Justicia y Derechos Humanos, Quito, 2009 , p. 43. 
nación -donde se supone que se continúan tomando, a partir de una base democrática, las decisiones que permiten el despliegue de la vida de los ciudadanos ${ }^{7}$. Se produce una desvinculación entre territorio, poder y mercado, de manera que el acceso al capital y a la tecnología, que permiten el despliegue de las funciones tradicionales del estado, ya no dependen tanto del control de una determinada porción de territorio como de las sinergias con quienes controlan las redes globales de producción de bienes y distribución de capitales ${ }^{8}$.

Efectivamente, «en un mundo "cableado" toda perturbación se transmite rápidamente a otros mercados y sociedades, ramificando los efectos del cambio», las posibilidades de la política en el sentido en que se ha entendido en los últimos siglos en Occidente son ciertamente limitadas. ${ }^{9}$. Progresivamente, la gramática del poder transita desde la legitimidad democrática anclada en la comunidad política en que se funda el estado-nación hacia la legitimidad posesiva - en términos monetarios- en que se traduce la disponibilidad de recursos en el proceso de acumulación capitalista ${ }^{10}$.

${ }^{7}$ Parto de la asunción de que, desde el punto de vista de la fundamentación del poder en el estado moderno constitucional que acompaña las fases anteriores del proceso de acumulación capitalista, existe una conexión fundamental entre la idea de individuo autónomo, fundamento de la comunidad política, y la idea de participación de democrática. En este sentido, por ejemplo, vid. CHEVALLIER, J., «Vers un droit post-moderne? Les transformations de la régulation juridique», Revue de Droit Public n. 3, 1998, p. 667. Ello se ve claramente en el famoso discurso de Gettysburg, pronunciado por Abraham Lincoln el 19 de noviembre de 1863, que termina con las palabras siguientes: «and that government of the people, by the people, for the people, shall not perish from the earth». Así, aunque muchos estados no funcionan realmente como regímenes democráticos-de hecho, algunos ni tan siquiera funcionan como auténticos estados-, lo cierto es que la legitimación democrática es el fundamento de la justificación del estado como tal. En este sentido, hay quién considera, incluso, que el futuro de «las instituciones democráticas que hoy conocemos» dependerá de que sobreviva el estado (vid. Sotelo, I., «La crisis del Estado», en Alcina Franch, J., y CAlÉs Bourdet, M. (Eds.), Hacia una ideología para el siglo XXI. Ante la crisis civilizatoria de nuestro tiempo, Akal, Tres Cantos, 2000, p. 229).

${ }^{8}$ Vid. Evans, P., «¿El eclipse del Estado? Reflexiones sobre la estatalidad en la época de la globalización», cit., p. 44.

9 Vid. Held, D., «¿Hay que regular la globalización? La reinvención de la política», en Carbonell, M., y VÁzQuez, R. (Eds.), Globalización y Derecho cit., p. 78.

${ }^{10}$ En este sentido, (las dificultades para) el acceso al capital por parte de los estados determinan las decisiones políticas internas, de modo que la legitimidad democrática de las decisiones queda gravemente erosionada. Un síntoma claro de ello es, en el caso de España, la reciente Ley Orgánica 2/2012, de 27 de abril, de Estabilidad Presupuestaria y Sostenibilidad Financiera (BOE, de 30 de abril de 2012), y, en particular, su artículo 14, en el que se prioriza el servicio de la deuda en el conjunto del gasto público. Así, los mercados condicionan las políticas públicas a través de instrumentos de presión distintos del voto democrático -la cotización de la moneda, el acceso al capital-, poniendo de manifiesto una nueva gramática del poder diferente de la que se sostiene formalmente -el fundamento democrático del poder-, basada en la disponibilidad de dinero, la forma abstracta del capital. Vid., en este sentido, EvANS, P., «¿El eclipse del Estado? Reflexiones sobre la estatalidad en la época de la globalización» cit., pág. 46. Sobre la naturaleza del dinero como 
Dicho proceso se articula, en la actualidad, a través de redes globales de comunicación que permiten el movimiento instantáneo de capital, sin que las fronteras de los estados puedan ponerle coto. Así, una masa de capital, que se autoalimenta, se halla en movimiento en la red global de los mercados, adquiriendo unas dimensiones que impiden que ninguna autoridad constituida de acuerdo con los parámetros clásicos del constitucionalismo - esto es, basada, directa o indirectamente, en el estado-nación- pueda contrarrestarla ${ }^{11}$.

Por otra parte, asimismo, las posibilidades tecnológicas permiten la fabricación de productos en múltiples espacios «nacionales», de modo que, también aquí, el proceso escapa, en parte, a las posibilidades de los estados, que se ven condicionados por la existencia de esta estructura global a la hora de articular sus políticas públicas ${ }^{12}$. En este sentido, la cohesión de la comunidad política depende de decisiones que toman actores privados en dicha estructura reticular, las cuales determinan tanto el acceso al capital que debe financiarla, como la inclusión en las redes globales de producción que permiten a la población adquirir bienes y servicios en el mercado global ${ }^{13}$. Los inversores devienen, en este contexto, los nuevos votantes, determinando más allá del principio «un hombre, un voto», la realización de políticas en todos los niveles de la estructura reticular del poder global, que trasciende el locus tradicional del poder político ${ }^{14}$.

\section{EL (¿NUEVO?) PAPEL DEL ESTADO-NACIÓN COMO ESTRUC- TURA INSTITUCIONAL DEL PROCESO DE ACUMULACIÓN CA- PITALISTA}

En el contexto descrito de desajuste entre el poder delimitado de los estados-nación y el flujo de recursos del mercado global, tienden a aparecer es-

expresión simbólica de la capacidad de consumo de bienes y servicios -medida de bienestar y de poder-, continua siendo interesante el análisis de Georg Simmel (1858-1918) en su Philosophie des Geldes. Para la aproximación de Simmel a esta cuestión, vid. FrisBY, D., Fragmentos de la modernidad. Teorías de la modernidad en la obra de Simmel, Kracauer y Benjamin, Visor, Madrid, 1992, pp. 162 ss.

${ }^{11}$ Vid. ReVELLI, M., «La ideología de la globalización y su realidad», en CARBONELL, M., y VÁzQuez, R. (Eds.), Globalización y Derecho cit., p. 126. Así, puede considerarse que «el Estado ha perdido poder frente a mutadas instituciones privadas que le estaban subordinadas», como señala JARA GóMEZ, A. M., en «Agentes transnacionales y eficacia horizontal de los derechos humanos. Una reflexión entorno a la demanda de Hazel Tau contra Glaxosmithkline y Boehringer Ingelheim», Teoría y Derecho n. 3, 2008, p. 328.

${ }^{12}$ Debe destacarse la existencia de redes de hardware, que permiten el despliegue de la fábrica global y la llamada «deslocalización de la producción». Vid., en relación con ello, BARredA, A., «Geopolítica, recursos estratégicos y multinacionales», Pueblos. Revista de información y debate, 2005, <http://www.revistapueblos.org/spip.php?article311> (última consulta realizada el 29 de mayo de 2012).

${ }^{13}$ Vid. Evans, P., «¿El eclipse del Estado? Reflexiones sobre la estatalidad en la época de la globalización» cit., p. 44.

${ }^{14}$ Vid. HeLD, D., «¿Hay que regular la globalización? La reinvención de la política» cit., p. 78. 
tructuras de poder informales y opacas, que dirigen, de algún modo, los procesos que determinan la circulación de los capitales y la generación de bienes y servicios, de los que depende, en última instancia, la vida de los seres humanos en todo el planeta, sin que se hayan generado procedimientos de control y limitación de dichos poderes, ni se haya alineado la exposición al poder global con una ciudadanía global. En este contexto, las personas son vulnerables ante diferentes amenazas -algunas que habían sido exorcizadas en la tradición constitucional, otras completamente nuevas-.

Sin embargo, el estado-nación, a pesar de todo, no ha desaparecido, sino que, como he de intentar mostrar en este apartado, continúa siendo un elemento esencial en la estructura reticular del poder en la que se despliega el mercado global, a pesar del discurso en relación con su superación que parece ir aparejado a la ideología del llamado consenso de Washington, en el marco del proceso de integración de los mercados que genera la situación actual de desajuste entre poder y control ${ }^{15}$. Efectivamente, el sistema interestatal es la pieza esencial de la infraestructura económica, que permite la existencia de los soportes físicos para el despliegue de las redes globales, así como asegurar el intercambio de bienes, servicios y capitales, tanto desde el punto de vista jurídico -el Derecho privado y el Derecho penal que garantizan la fiabilidad de las transacciones, así como el aparato judicial que constituye su apoyo último-, como desde el punto de vista estrictamente físico -las estructuras policiales y militares que defienden las estructuras globales de dominación- $-^{16}$.

${ }^{15}$ Sobre el Consenso de Washington, vid. Williamson, J., «A Short History of the Washington Consensus», ponencia presentada en el Congreso From the Washington Consensus towards a new Global Governance, que tuvo lugar en Barcelona, los días 24 y 25 de septiembre de 2004, <http://www.iie.com/publications/papers/williamson0904-2.pdf> (última consulta realizada el 29 de mayo de 2012).

${ }^{16}$ En cuanto a lo primero, vid., una vez más, Evans, P., «¿El eclipse del Estado? Reflexiones sobre la estatalidad en la época de la globalización» cit., pp. 45 y 52 . En cuanto a lo segundo, es evidente que los estados constituyen la estructura básica a partir de la que se llevan a cabo las acciones armadas destinadas a garantizar el flujo de recursos a nivel global en el marco de la estructura global de dominación derivada de la integración de los mercados. El caso más claro es la llamada 'Guerra contra el Terror', en que los perdedores del sistema son combatidos por los aparatos militares de los estados del Norte y, particularmente, de la potencia hegemónica, para asegurar, entre otras cosas el acceso a los recursos naturales que alimentan el mercado global. En este contexto, el terrorismo se presenta como una consecuencia del abismo de bienestar entre los países desarrollados y aquéllos en vías de desarrollo, tal como subraya JonAs, H., en El principio de responsabilidad. Ensayo de una ética para la civilización tecnológica, Herder, Barcelona, 1995, p. 294. Sobre el papel de los estados en la defensa de la estructura global del poder y del aseguramiento del flujo de los recursos hacia los centros de poder, especialmente, en el caso de África, vid. Fontana, J., Por el bien del imperio. Una historia del mundo desde 1945 cit., pp. 707 ss. En relación con el mismo proceso en el caso del Oriente Medio, vid. FISK, R., The Great War for Civilisation. The Conquest of the Middle East, Harper, LondresNueva York-Toronto-Sidney, 2006. 
En este contexto, el sistema de estados-nación deviene una estructura imperial, en que, oculto por el velo que ofrece el mito westfaliano de la soberanía y la igualdad entre los estados, se plantean unas relaciones extremadamente desiguales ${ }^{17}$. Así, unos estados ocupan el centro y aseguran el espacio para el despliegue, en condiciones más o menos seguras, del modelo de vida de los ganadores del sistema, mientras que otros se sitúan en la periferia, deviniendo sencillas estructuras parciales de dominación, dirigidas al control social de los perdedores del sistema y al aseguramiento del flujo de los recursos hacia el centro ${ }^{18}$. En este contexto, una estructura jurídica, articulada, en buena parte, al entorno de la OMC y el GATT, se impone sobre los estados determinando la permanencia del intercambio desigual de recursos y la progresiva desprotección ante las estructuras globales de poder (e intercambio) de una cantidad muy numerosa de miembros de la comunidad global ${ }^{19}$.

De este modo, los estados-nación aseguran, en su conjunto, el funcionamiento de la economía global, aunque sometidos a los flujos de capitales, bienes y servicios, que facilitan, pero no pueden controlar. En este sentido, es evidente

${ }^{17}$ Sobre la disolución de la idea moderna de soberanía absoluta en la complejidad del mundo contemporáneo, vid. Llano, A., La nueva sensibilidad, Espasa, Madrid, 1988, p. 121.

${ }^{18}$ Sobre la idea de centro y periferia en el funcionamiento del sistema-mundo capitalista, vid. TAYLOR, P. J., y Flint, C., Geografía política. Economía-mundo, estado-nación y localidad, Trama, Madrid, 2002 ${ }^{2}$, pp. 21 ss. Esta idea fue desarrollado en primera instancia en el ámbito de Comisión Económica para América Latina (CEPAL) por economistas como el argentino Raúl Prebisch, que publicó en 1949 el trabajo «Crecimiento, desequilibrio y disparidades: interpretación del proceso de desarrollo económico», dentro del Estudio económico de América Latina, editado por la citada organización. A partir de ese momento, este patrón hermenéutico de los flujos globales de recursos se consolida con aportaciones como la del sueco Gunnar Myrdal, que subraya la imposibilidad de igualación espontánea por el hecho de que el capital (recursos económicos) y el trabajo (mano de obra cualificada) son atraídos hacia los espacios donde se ha construido una ventaja inicial (el centro). En relación con ello, vid. ConTI, S., Geografia economica. Teorie e metodi, Turín: UTET, 1996, pp. 129 ss. En la teoría neomarxista, se subraya que el subdesarrollo (la condición de periferia) acaba siendo consustancial a la generación de la economía global, ya que, de hecho, permite superar las contradicciones en el centro a partir del aseguramiento del intercambio desigual de recursos fundado en la posición predominante de las comunidades hegemónicas. Vid., en relación con ello, op. cit., pág. 166. Aunque es cierto que el carácter absoluto de la partición entre centro y periferia debe matizarse con las aportaciones de la teoría de sistemas (op. cit., p. 226), lo cierto es que tiene un importante potencial explicativo en la lectura de las estructuras de poder del presente.

${ }^{19}$ Es lo que Clarkson, S., y Wood, S., en A Perilous Imbalance. The Globalization of Canadian Law and Governance, UBC Press, Vancouver-Toronto, 2009, particularmente pp. 161 ss., han denominado «Supraconstitución», esto es, fundamentalmente, las reglas de la economía global que se imponen sobre las decisiones de los estados incluso a nivel constitucional, lo que es particularmente importante en los estados de la periferia. Sobre las relaciones de intecambio desigual y el drenaje de recursos de la periferia hacia el centro, vid. Conti, S., Geografia economica. Teorie e metodi cit., pp. 173 ss. 
que no hay mercado sin sistema jurídico, sin aparato institucional que garantice el flujo seguro de los bienes y servicios, dentro de un determinado esquema normativo, aunque ello no disminuye la vulnerabilidad del estado a la hora de intentar perseguir una agenda interior propia ante las imposiciones de las estructuras globales de poder e intercambio ${ }^{20}$. Sea como sea, las estructuras estatales son imprescindibles en el funcionamiento de la economía global, que, dado que genera un flujo desigual de recursos, está necesitada, asimismo, del ejercicio de la violencia legítima por parte de los estados para proteger la asignación de los recursos ante aquellos que la impugnan, a parte de los marcos reguladores que estos también proporcionan.

En este contexto, los estados-nación cumplen sus funciones de acuerdo con el lugar que ocupan en el sistema-mundo capitalista, esto es, el centro o la periferia. En el primer caso, realizan funciones características del estado-nación tradicional en relación con la garantía de la seguridad (jurídica y física) interior, que se proyecta sobre la vida cotidiana de los que habitan dentro de sus fronte$\mathrm{ras}^{21}$. En el segundo caso, en cambio, devienen estructuras de dominación, diseñadas para permitir el acceso a los recursos a actores externos, más que para garantizar, en algún sentido, las condiciones de vida de los actores internos ${ }^{22}$.

En este sentido, es importante observar como los estados del centro actúan como garantes del funcionamiento de la economía global y el flujo desigual de recursos en dos sentidos, relacionados con las tradicionales funciones de policía que el liberalismo clásico asignaba a los poderes públicos ${ }^{23}$. Ello

\footnotetext{
${ }^{20}$ Vid. LAPORTA, F. J., «Globalización e imperio de la ley. Algunas dudas westfalianas», en CArbonell, M., y VÁzQuez, R. (Eds.), Globalización y Derecho cit., pp. 213 ss.

${ }^{21}$ Solo en este contexto, se entiende la afirmación del papel de los estados contemporáneos como espacios democráticos y garantes de los derechos. Vid., por ejemplo, THÜRER, D., «Recht der internationalen Gemeinschaft und Wandel der Staatlichkeit», en THÜRER, D., Aubert, J.-F., y Müller, J. P. (Eds.), Verfassungsrecht der Schweiz / Droit constitutionnel suisse, Schulthess, Zúrich, 2001, p. 55.

${ }^{22}$ No debe perderse de vista el carácter desigual del flujo de los recursos en la economía global, lo que conduce a unas estructuras de poder reales que, de hecho, intentan asegurar la continuidad de las relaciones centro-periferia, lo que asigna un papel determinado a los estados periféricos, cuya posibilidad de articular políticas autónomas de desarrollo queda francamente comprometida. En relación con el flujo desigual de los recursos, vid. AlTVATER, E., El precio del Bienestar, Alfons el Magnànim, Valencia, 1994, p. 188; y Ridoux, N., Menos es más. Introducción a la filosofia del decrecimiento, Los libros del lince, Barcelona, 2009, p. 32. Para una recopilación de datos que muestran dicha desigualdad, vid. DAVIES, J. B., SANDSTROM, S., SHORROCKS, A., y WolfF, E. N., The World Distribution of Household Wealth <http://www.iariw. org/papers/2006/davies.pdf $>$ (última consulta realizada el 29 de mayo de 2012).

${ }^{23}$ Efectivamente, el aseguramiento, a través de la violencia (legítima) si es necesario, de la propiedad sobre los recursos y la fiabilidad de los intercambios, es una función tradicional del Estado que, nuevamente, desempeña en el momento actual, como guardián de la economía global. Sobre la caracterización de tal función en cuanto a fundamental en el estado como institución moderna, vid. CrooK, S., PATUlski, J., y Waters, M., Postmodernization. Change in Advanced Society, Sage, Londres-Thousand Oaks-Nueva Delhi, 1992, p. 14.
} 
puede verse en relación con el combate contra el terrorismo global, que justamente aparece como impugnación de las estructuras globales de dominación, a partir de parámetros culturales generados en el mundo islámico, que, particularmente, ofrece una alternativa significativa a los perdedores del sistema $^{24}$. Por otra parte, debe subrayarse el papel de los estados a la hora de asegurar la impenetrabilidad de sus fronteras. La tecnología que facilita el tránsito de los capitales y los bienes, así como el marco jurídico que lo permite, no alcanzan a las personas, que se ven impedidas de buscar oportunidades en una economía global pretendidamente abierta gracias a los controles fronterizos de los estados del centro, de modo que «cuanto más se hacen suaves y permeables al movimiento del dinero, tanto más esas mismas líneas de frontera se hacen duras e impenetrables al movimiento de los hombres» ${ }^{25}$.

Las distancias físicas, efectivamente, se reducen, pero en cambio las desigualdades aumentan ${ }^{26}$. En consecuencia, también lo hacen las necesidades de controlar los flujos de población, para asegurar, en última instancia, el acceso desigual a los recursos en el marco de las reglas de la economía global -conjunto complejo que agrupa normas «nacionales», el marco normativo de la $\mathrm{OMC}$, las determinaciones de las instituciones financieras internacionales y la llamada nova lex mercatoria-. El papel de los estados-nación es determinante en este contexto, de modo que puede decirse que el «peligro no es que los Estados terminen como instituciones marginales, sino que formas más limitadas y represivas de organizar el papel del Estado sean aceptadas como la única manera de evitar el colapso de las instituciones públicas» ${ }^{27}$.

En definitiva, las estructura globales de poder que garantizan el flujo de recursos y la circulación de los capitales son, en parte, opacas, y se imponen a las decisiones democráticas que afloran en el marco político-jurídico del estado-nación, que pasa a jugar un papel ancilar en relación con ellas. Ello no hace menos importante ese papel en el funcionamiento de la red global de poder, pero, en todo caso, lo sitúa en una posición subordinada y, en muchas

${ }^{24}$ De ello, probablemente, se deriva el atractivo del islamismo en África. En relación con la perspectiva alternativa que supone el Islam sobre la organización social y, en especial, sobre la cuestión de los derechos, poniendo el énfasis en la protección del bienestar, más que en la protección de la autonomía, vid. GlenN, H. P., Legal Traditions of the World, Oxford University Press, Oxford-Nueva Cork, $2007^{3}$, pp. 212 ss. Sobre la naturaleza del terrorismo de origen islamista, vid. JORDÁN, J., «Terrorismo yihadista y Estado de Derecho», Teoría y Derecho n. 3, 2008, pp. 20-33.

${ }^{25}$ Vid. Revelli, M., «La ideología de la globalización y su realidad» cit., p. 128. De hecho, el capital se presenta como más móvil que la población en la configuración de la economía global. Vid., en este sentido, ConTI, S., Geografia economica. Teorie e metodi cit., p. 160.

${ }^{26}$ Vid. Toscano, R., «Interrogantes éticos sobre la globalización», en CARBONELL, M., y VÁzQuez, R. (Eds.), Globalización y Derecho cit., p. 98.

${ }^{27}$ Vid. Evans, P., «¿El eclipse del Estado? Reflexiones sobre la estatalidad en la época de la globalización» cit., pág. 27. 
ocasiones, contradictoria con las finalidades que le asigna la tradición constitucional, fundamentalmente, en la periferia del sistema ${ }^{28}$.

Si tomamos en serio el compromiso del constitucionalismo con el control del poder y el aseguramiento del status de las personas, parece clara la necesidad de una transformación, más allá del estado, que permita afrontar el nuevo (des)equilibrio de poderes planteado por la integración de los mercados, afrontando las estructuras reales del poder global y partiendo de una idea inclusiva de ciudadanía en el marco de una sociedad globalmente integrada, pero profundamente injusta. La cuestión, por lo tanto, no es la disolución del sistema jurídico y del aparato institucional, si no más bien, la generación de un estructura institucional global que ofrezca garantías en relación con dos aspectos clave e interdependientes: el control del poder y el status de los miembros de la comunidad.

\section{TECNOLOGÍA, VELOCIDAD Y FRAGMENTACIÓN. UNA NUEVA DIVISIÓN DE PODERES}

Empecemos por el control del poder, un poder difuso y, aparentemente, inarticulado, líquido, que, utilizando el hardware que proporcionan, mayormente, los estados del centro - las infraestructuras que permiten la circulación de información y bienes, financiadas a través de los impuestos que aportan los ciudadanos de dichos estados-, se proyecta a nivel global, condicionando las decisiones de esos mismos estados y laminando el contenido de la democracia. En este contexto, uno de los factores más disfuncionales del proceso de acumulación capitalista en su fase actual es la velocidad de circulación de la información, que permite la especulación, desestabiliza los marcos democráticos y compromete la cohesión social ${ }^{29}$.

\footnotetext{
${ }^{28}$ En cierto modo, hay quien plantea el ocaso del estado-nación, sobre todo, si nos concentramos en la literatura jurídico-constitucional, desde el punto de visto, de la cesión de poderes hacia estructuras institucionales supra o infraestatales. En este sentido, CANO BUESO, J., en «Algunas cuestiones centrales del proceso de globalización», en BALAGUER CALLEJón, F. (Coord.), Derecho constitucional y cultura. Estudios en homenaje a Peter Häberle, Tecnos, Madrid: 2004, p. 189, considera que el Estado-nación «se evapora hacia arriba (como sucede con la construcción de la Unión Europea) o se disuelve hacia dentro a la búsqueda de microespacios donde el ciudadano puede controlar y decidir su propio espacio vital, como sucede, sin ir más lejos, con la potenciación de las regiones y de las ciudades». Para una opinión algo más matizada, pero de sentido similar, vid. ARBós, X., y Vernet I LlOBET, J., «Los nuevos federalismos y el constitucionalismo del siglo XXI», en APARICIO, M. A. (dir.), La Descentralización y el Federalismo. Nuevos modelos de Autonomía Política (España, Bélgica, Canadá, Italia y Reino Unido), Barcelona, Cedecs, 1999, pp. 261-271. Sin embargo, por una parte, continua siendo cierto que el estado-nación juega un rol central, tanto en relación con la toma de decisiones (si bien condicionada) en el interior, como en relación con la estructura global del poder. Vid., en este sentido, Clarkson, S., y Wood, S., A Perilous Imbalance. The Globalization of Canadian Law and Governance cit., p. 222.

${ }^{29}$ Así, vid. CARBonell, M., "Globalización y derecho: algunas coordenadas para el debate», en CArbonell, M., y VÁzquez, R. (Eds.), Globalización y Derecho cit., p. 27.
} 
Existe una vinculación entre la interconexión de los flujos de información, la extensión mundial de los intercambios de mercancías y deslocalización de los procesos productivos, que acaba generando estructuras de poder más allá del estado-nación poco transparentes, poco susceptibles al control y poco legitimadas desde el punto de vista democrático ${ }^{30}$. En este sentido, la velocidad con la que se llevan a cabo, a través de las redes de soporte de la transmisión de la información, las operaciones de transmisión de capitales de gran escala acaba generando una gran volatilidad que se proyecta sobre la cohesión de las comunidades políticas y genera desigualdades e inseguridades en la escala global en que se producen dichos movimientos ${ }^{31}$.

Se plantean aquí los dos grandes problemas del funcionamiento del metabolismo social global, que se corresponde con un sistema institucional más complejo y dinámico que el estado-nación tradicional, que, sin embargo, como hemos visto, forma parte de él. En relación con el status de los miembros de la comunidad global, debe señalarse que el acceso a los beneficios derivados del uso de los recursos naturales aparece como claramente injusto, generándose no solo desigualdades difícilmente justificables, sino incluso situaciones manifiestas de exclusión, que, por otra parte, parecen aumentar y no disminuir a medida que se despliega con mayor plenitud la estructura de dominio correspondiente a la integración de los mercados ${ }^{32}$. La universalidad de los flujos no se corresponde, en definitiva, con el acceso equitativo al producto del metabolismo social.

En este sentido, la movilidad de los capitales a través de las nuevas redes generadas por las tecnologías de la información y la comunicación da lugar a la especulación y a una asignación de recursos ciertamente discutible a nivel global. Si la legitimidad de un mercado global que sustituyera a las estructuras tradicionales de poder se basa en una mejora de las condiciones de vida de los miembros de la comunidad global, parece que, más bien, el modelo está fracasando. De este modo, desde un punto de vista constitucional, no se proporciona una inclusión adecuada de los miembros de la comunidad global en las nuevas estructuras de poder -en términos de participación tanto como de bienestar-, ni se les protege adecuadamente ante las intromisiones de este nuevo poder global en sus vidas.

${ }^{30}$ Vid. Bovero, M., «¿Siete globalizaciones?», en Carbonell, M., y VÁzQuez, R. (Eds.), Globalización y Derecho cit., p. 136.

${ }^{31}$ Vid. Evans, P., «¿El eclipse del Estado? Reflexiones sobre la estatalidad en la época de la globalización» cit., p. 52.

${ }^{32}$ En este sentido, Paul Krugman ha hablado de la «gran divergencia». Vid. FonTANA, J., Por el bien del imperio. Una historia del mundo desde 1945 cit., p. 565. En relación con el progresivo aumento de las desigualdades entre los seres humanos y las diferentes comunidades en un contexto global, vid., asimismo, MARGALEF, R., «Lo que se llama ecología y posibles condicionantes de nuestro futuro», en AlcINA Franch, J. y CALÉs BourDET, M. (Eds.), Hacia una ideología para el siglo XXI. Ante la crisis civilizatoria de nuestro tiempo cit., p. 330. 
La misma facilidad con que circula la información y, a través de ella, los capitales, cuya permanencia en un determinado lugar, por decirlo de algún modo, puede ser fugaz, da lugar a la consolidación de poderes informales, generalmente opacos y de carácter líquido, que condicionan la vida de los miembros de la comunidad global sin que sea posible, en la mayoría de los casos, exigirles responsabilidades, así como tomar parte en sus decisiones. En este contexto, es donde aparece la necesidad de controlar y delimitar el poder, de acuerdo, por otra parte con la tradición constitucional, que debe desplegarse en un nuevo escenario.

Es pertinente aquí señalar la importancia de la separación de poderes en dicha tradición a la hora de articular procedimientos de control del poder, cuya finalidad última, en definitiva, es la garantía del status de los miembros de la comunidad política ${ }^{33}$. Se impone, según mi criterio, un sistema de checks and balances más eficaz y transparente en el diseño de las estructuras globales de poder, que, particularmente, debe fundarse en la fragmentación y la diversidad para generar barreras jurídico-políticas al flujo de datos y, de este modo, poner al servicio de la sociedad en su conjunto los recursos generados en el sistema, evitando las tendencias destructivas implícitas en la multiplicación de las maniobras especulativas que la actual estructura desregulada de la red permite.

En relación con esto, debe subrayarse, asimismo, que la implantación del sistema-mundo capitalista a nivel global constituye una amenaza para la diversidad cultural ${ }^{34}$. Ello no solo es un problema desde el punto de vista utili-

${ }^{33}$ En este sentido, vid., por ejemplo, Torres del Moral, A., «Naturaleza jurídica de los derechos constitucionales», en Balaguer Callejón, F. (Coord.), Derecho constitucional y cultura. Estudios en homenaje a Peter Häberle cit., p. 507.

${ }^{34}$ En este sentido, es evidente que el proceso de integración de los mercados y la expansión del sistema-mundo capitalista han significado la progresiva marginación de las culturas externas a la civilización hegemónica. Vid., en este sentido, Estermann, J., Filosofía andina. Estudio intercultural de la sabiduría autóctona andina, Abya Yala, Quito, 1998, p. 33; SÁNCHEZ, C., «Autonomía y pluralismo. Estados plurinacionales y pluriétnicos», en GonzÁlez, M., Burguete Cal y Mayor, A., y Ortiz-T., P., (Eds.), La autonomía a debate. Autogobierno indígena y Estado plurinacional en América Latina, FLACSO (Sede Ecuador) / Cooperación Técnica Alemana (GTZ) / Grupo Internacional de Trabajo sobre Asuntos Indígenas (IWGIA) / Centro de Investigaciones y Estudios Superiores en Antropología Social (CIESAS) / Universidad Intercultural de Chiapas (UNICH), Quito, 2010, p. 281; TORRES GALARZA, R., «Diversidad y globalización: los derechos en su laberinto», en LEFF, E. (Coord.), Justicia Ambiental: Construcción y Defensa de los Nuevos Derechos Ambientales Culturales y Colectivos en América Latina, Programa de las Naciones Unidas para el Medio Ambiente / Universidad Nacional Autónoma de México, México DF. 2001, p. 69; WALSH, C., «Interculturalidad, plurinacionalidad y decolonialidad: las insurgencias político-epistémicas de refundar el Estado», Tabula Rasa n. 9, 2008, p. 137. En cierto modo, puede decirse que ha habido un esfuerzo dirigido a promover la homogeneización cultural, con estrategias de banalización de la diferencia, como pone de manifiesto MORENO NAVARRO, I., en «Quiebra de los modelos de modernidad, globalización e identidades colectivas», en Alcina Franch, J., y CAlÉs Bourdet, M. (Eds.), Hacia una ideología para el siglo XXI. Ante la crisis civilizatoria de nuestro tiempo cit., p. 107. 
tario de la comunidad global -empobrecimiento en relación con conocimientos y tradiciones cuya virtualidad puede ser importante en el futuro-, sino que también es manifiestamente discutible desde el punto de vista del respeto a las personas, ya que dicha amenaza se cierne sobre el proyecto de vida de una cantidad muy importante de miembros de la comunidad global, erosionando su dignidad y generando exclusión ${ }^{35}$.

La diversidad cultural - como la diversidad biológica- constituye una barrera consistente ante el flujo descontrolado de información, tendiendo a generar espacios de protección y a limitar los efectos destructivos de la especulación ${ }^{36}$. A partir de aquí, es imaginable un sistema institucional global donde las diferentes comunidades culturales articulan espacios normativos diversos, fragmentando territorialmente las reglas del juego y sometiendo a los flujos globales al control de estructuras democráticas locales que deben funcionar como impedimentos para la acumulación del poder, así como elementos para su fijación y la exigencia de responsabilidad en su ejercicio ${ }^{37}$.

En mi opinión, antes que tender hacia un espacio normativo homogéneo que se corresponda con el mercado global -un imaginario gobierno global, parece adecuado fragmentar los espacios políticos, de modo que los centros opacos de poder encuentren límites análogos a la idea de checks and balances del federalismo norteamericano ${ }^{38}$. Por decirlo con Roberto Toscano, no se pretende «un hipotético (y si no fuera hipotético, peligroso por lo que respecta a la diversidad y al pluralismo) «Gobierno mundial», sino más bien (...) una

${ }^{35}$ Así lo he defendido en «Circles of Consensus. The Preservation of Cultural Diversity through Political Processes», Utrecht Law Review n. 8-1, 2012, especialmente, pp. 94 ss.

${ }^{36}$ Sobre la analogía entre diversidad cultural y diversidad biológica, vid., por ejemplo, Moreno NAVARRo, I., «Quiebra de los modelos de modernidad, globalización e identidades colectivas» cit., p. 129; Moulines, U., «Assaig d'una teoria (semi-)formal de les nacions», Quaderns de filosofia i ciència n. 36, 2006, p. 16; Velasco, J. C., «El Multiculturalismo, ¿una nueva ideología? Alcance y límites de la lucha por las identidades culturales», en ALCInA Franch, J., y Calés Bourdet, M. (Eds.), Hacia una ideología para el siglo XXI. Ante la crisis civilizatoria de nuestro tiempo cit., p. 151.

${ }^{37}$ Nuevamente, vid. mi trabajo, «Circles of Consensus. The Preservation of Cultural Diversity through Political Processes» cit., p. 97 ss.

${ }^{38}$ En este sentido, debo remitirme al texto clásico de James Madison en los Federalist Papers. He utilizado la edición catalana: MAdison, J., HAMILTON, A., y JAY, J., El Federalista, Institut d'Estudis Autonòmics, Barcelona, 2009, pp. 430 ss. La idea de la separación de poderes como garantía del status de los ciudadanos se desarrolla junto con la propia tradición constitucional. En este sentido, deben mencionarse las obras clásicas de LOCKE, J., Segundo Tratado sobre el gobierno civil, Alianza, Madrid, 1990; y MoNTESQUIEU, Ch.L. de Secondat, barón de, Del espíritu de las leyes, Tecnos, Madrid, 1985. A partir de está matriz, se entiende que no hay libertad sin separación de poderes, como pone de manifiesto RAMírez JimÉnEZ, M., «De las relaciones entre el Gobierno y las Cortes Generales. Comentario introductorio al Título V», en Alzaga VillaAmil, Ó. (Dir.), Comentarios a la Constitución española de 1978 (VIII), Edersa, Madrid, p. 672. En un sentido análogo, vid. Aragón, M., «Constitución y derechos fundamentales», en CARbonell, M., (Comp.), Teoría de la Constitución. Ensayos escogidos, Porrúa, México D. F., 2005, p. 218. 
multiplicación de niveles de gobierno (...), por un lado, y de la introducción de elementos de transnacionalidad relacionados con una situación individual o de grupo, por otro ${ }^{39}$.

Se trata, en definitiva, de fragmentar la democracia, creando barreras para el flujo descontrolado de información. En este sentido, se deberían tender hacia la construcción de ámbitos diferentes y solapados, vinculados todos más o menos directamente a la idea de democracia -quizá a las ideas de democracia- en algunos de los espacios básicos - la ciudad, el poblado, la tribu, la nación, etc.-- Con esta idea de círculos de consenso diferenciados, superpuestos y mutuamente vinculados, me parece obvio que las estructuras globales de poder serían reconducidas hacia una limitación de sus posibilidades que redundaría en beneficio de los individuos y colectivos de la comunidad global.

Por otra parte, asimismo, se preservaría la diversidad, siendo ambas cosas imprescindibles para la construcción de un status de los miembros de la comunidad global que se corresponda con una idea contemporánea de dignidad humana, correspondiente a la situación de hecho que la realidad tecnológica y los condicionantes impuestos por el carácter limitado y vulnerable de los recursos naturales ${ }^{40}$. Por otra parte, se conseguiría una cierta flexibilidad en relación con la respuesta de las comunidades de decisión -las democracias fragmentadas e interconectadas- ante la geografía variable de los problemas contemporáneos ${ }^{41}$.

Dichas democracias fragmentadas deberían integrarse en espacios políticos más amplios, de acuerdo con la idea de constitucionalismo multinivel, donde se combinarían las particularidades culturales de las diferentes comunidades, definidas de acuerdo con sus propias singularidades identitarias, con la necesidad de reglas de juego progresivamente más comprensivas, hasta el nivel global, fundado en una idea de derechos humanos que debería ser abierta al diálogo intercultural y ajustada a la disponibilidad de recursos. Para los miembros de la comunidad global ello representaría el disfrute de un status de ciudadanía múltiple, en el que se definirían dife-

${ }^{39}$ Vid. Toscano, R., «Interrogantes éticos sobre la globalización», en CARBONELL, M., y VÁzQuez, R. (Eds.), Globalización y Derecho cit., p. 105.

${ }^{40}$ Creo que continua siendo relevante la idea de dignidad humana como fundamento del orden constitucional. Ello ha sido así en el caso del constitucionalismo de matriz estatal desde el final de la Segunda Guerra Mundial. Vid., por ejemplo, BENDA, E., «El Estado social de Derecho», en Benda, E., Mainofer, W., Vogel, H.-J., Hesse, K., Heide, W., Manual de Derecho Constitucional, Marcial Pons, Madrid, 2001, pp. 491-492; ENÉRIZ OlAECHEA, F. J., La protección de los derechos fundamentales y las libertades públicas en la Constitución Española, Universidad Pública de Navarra-Nafarroako Unibertsitate Publikoa, Pamplona, 2007, p. 154; WEBER-FAs, R., Der Verfassungsstaat des Grundgesetzes, Mohr-Siebeck, Tubinga, 2002, p. 79.

${ }^{41}$ Vid. FreY, B. S., «Gobierno flexible para un mundo globalizado», en en CARBONELL, M., y VÁzQuez, R. (Eds.), Globalización y Derecho cit., p. 179. 
rentes niveles de pertenencia relacionados con elementos particulares de dicho status $^{42}$.

\section{DE DEMOCRACIAS FRAGMENTADAS A INSTRUMENTOS DE CONTROL GLOBAL}

Así como el espacio de la toma de decisiones -que se corresponde con el Estado democrático en el constitucionalismo tradicional- debe fragmentarse, como he expuesto, para limitar y fijar las nuevas formas de poder que afloran en el proceso de integración de los mercados, el espacio del control del poder, por su parte, debe integrarse, ahora sí, de acuerdo con la propia dinámica del mercado global, o aquello que, eventualmente, le sustituyera como metabolismo social planetario. La idea fundamental aquí es que, si partimos de la comunidad global como dato y lo que pretendemos es dotarla de instrumentos de gobernanza que, en última instancia, garanticen el status de sus miembros, debemos tener, por una parte, un núcleo de consenso general $\mathrm{y}$, por otra, parte instrumentos de control global que, reposando en el pluralismo político y cultural, sean capaces de garantizarlo ante los actores más poderosos del sistema ${ }^{43}$.

El control del poder debería corresponderse con el espacio del poder. Como este ha trascendido las barreras del estado-nación, al menos, parcialmente, y lo seguiría haciendo en el modelo de pluralismo superpuesto, democracia fragmentada y constitucionalismo multinivel, que aquí proponemos, ello nos lleva a la necesidad, correspondientemente, de procesos e instituciones de control global. Debemos reconducir las formas proteicas del poder a un sistema de control integrado, que sea expresión y garantía del consenso social fundamental a escala global ${ }^{44}$.

Con ello, extenderíamos, la idea de Estado de Derecho, vinculada a la garantía de status de los miembros de la comunidad política y a la limitación del poder, más allá del estado -también sería necesario hacerlo más acá-45. En este sentido, podría ser interesante la idea de comunidad de Derecho que Walter Hallstein propuso para Europa y que nos permitiría responder adecua-

${ }^{42}$ Sobre esta idea de ciudadanía múltiple, vid., por ejemplo, Held, D., «¿Hay que regular la globalización? La reinvención de la política» cit., p. 83.

${ }^{43}$ Sobre la relación entre el control y la protección de los derechos de los miembros de la comunidad política -lo que vengo designando como el status-, vid. BIZEAU, J.-P., «Pluralisme et démocratie», Revue de Droit Public n. 2, 1993, p. 538.

${ }^{44}$ Vid. Guastini, R., «La Constitución como límite a la legislación», en CARBonell, M. (Comp.), Teoría de la Constitución. Ensayos escogidos cit., p. 235.

${ }^{45}$ En este sentido, como recuerda MichaEL, L., en «El Estado constitucional cooperante», en Balaguer Callejón, F. (Coord.), Derecho constitucional y cultura. Estudios en homenaje a Peter Häberle cit., p. 328, «el principio Estado de Derecho está menos ligado a la estatalidad de lo que podría pensarse a tenor de su historia». 
damente tanto a la pluralidad de círculos de consenso, como a las necesidades de control en el nivel más elevado del sistema ${ }^{46}$.

Desde el punto de vista institucional, deberían pensarse en estructuras de tipo jurisdiccional -o quizá, sistemas más complejos que incluyesen instituciones de legitimidad fundada en la representación y legitimidad fundada en el conocimiento experto-. En cualquier caso, la misión de tales estructuras globales de control debería ser el garantizar que los diferentes actores del sistema global se comportan de acuerdo con los condicionantes derivados del consenso sustantivo planetario y de la diversidad de sistemas institucionales en presencia.

Las fundamentos sustantivos para el ejercicio del control a ese nivel deberían basarse en la cultura de los derechos humanos, como acervo fundamental de la tradición constitucional occidental con capacidad para generar reglas inclusivas apropiadas para construir y preservar un status mínimo, dentro de la comunidad global, para todos sus miembros, acorde con la dignidad de la persona ${ }^{47}$. Sin embargo, dicha apelación a los derechos humanos debería matizarse en un doble sentido. Por una parte, debería aceptarse el carácter dinámico del consenso en el marco de un diálogo intercultural serio, que permitiera aflorar las aportaciones de los acervos culturales distintos del hegemónico. Por otra parte, como hemos de ver en el siguiente apartado, la tradición de los derechos debería reformularse en términos de responsabilidad, de acuerdo con la aceptación pragmática de los límites que el carácter vulnerable y limitado de los recursos ponen a su satisfacción incondicionada.

\section{ROMPIENDO CON EL PROGRESO: ADAPTACIÓN AL MEDIOY RESPONSABILIDAD EN UN NUEVO CONSTITUCIONALISMO}

De la fragmentación del espacio de decisión y la integración del espacio de control, se deriva un constitucionalismo multinivel, reconstruido para responder a los problemas que plantea la integración de los mercados, tanto desde el punto de vista tradicional de la limitación del poder, como desde el punto de vista novedoso de la gestión de recursos vulnerables y limitados. En este

${ }^{46}$ En relación con ello, vid. STOLLeIS, M., «Europa como Comunidad de Derecho», Historia constitucional n. 10, 2009, pp. 475-485<http://www.historiaconstitucional.com> (última consulta realizada el 29 de mayo de 2012).

${ }^{47}$ En relación con la idea de los derechos humanos como fundamento y vehículo para el diálogo intercultural y el establecimiento de consensos a nivel global, el punto de vista que aquí defiendo entiendo que está en sintonía -aunque quizá va más allá- con el consagrado en la Convención sobre la protección y promoción de diversidad de las expresiones culturales de la UNESCO, particularmente, en su artículo 2 (cito a partir del documento oficial en castellano disponible en la siguiente página web: $<$ http://unesdoc.unesco.org/images/0014/001429/142919s.pdf $>$, última consulta realizada el 28 de noviembre de 2011). La idea de la dignidad igual de todas las culturas entiendo que debe ser el fundamento para el diálogo intercultural y la creación de un espacio político efectivamente compartido. 
sentido, como se habrá observado, he ido repitiendo la expresión «status» y he evitado cuidadosamente la expresión «derechos» para referirme a la posición jurídica fundamental de los miembros de la comunidad global que debería ser objeto de protección en el marco de un constitucionalismo multinivel como el que aquí se propone para responder a las amenazas que plantean las actuales estructuras de poder, desarrolladas a partir del proceso de integración de los mercados y las mejoras tecnológicas en el campo de la información y la comunicación.

Efectivamente, dada la situación de escasez y vulnerabilidad de los recursos naturales que alimentan el metabolismo global, según mi punto de vista debe romperse con la confianza utópica en un progreso permanente que siempre permita la satisfacción de unos derechos construidos a partir de una idea abstracta de individuo, para aceptar que los derechos en el futuro van a tener que ejercerse de forma limitada y, sobre todo, de acuerdo con el principio de responsabilidad, derivado de las posibilidades tecnológicas de que disponemos los seres humanos, que ponen en riesgo la pervivencia de la vida como la conocemos y, en particular, la posibilidad de las generaciones futuras de disponer de recursos para satisfacer sus necesidades ${ }^{48}$.

En este sentido, aceptando que el núcleo del consenso global debe fundamentarse en la tradición de los derechos humanos, creo que, asimismo, debe integrarse en él la idea de responsabilidad ${ }^{49}$. Ello debe vincularse con la noción de justicia ambiental -que debe garantizar el acceso equitativo a los beneficios derivados del uso de los recursos naturales tanto a los miembros actuales de la comunidad global como a las generaciones futuras, así como un reparto igualmente equitativo de las cargas-, así como un cierto respeto por la vida,

\footnotetext{
${ }^{48}$ Sobre la idea de la vulnerabilidad y la escasez de los recursos naturales, deben articularse las propuestas en la línea de generar una comunidad global auténticamente inclusiva, abandonando una confianza ciega en el progreso tecnológico y la competencia en los mercados como medios para llegar a ello. He desarrollado esta idea en mi trabajo «Environmental Justice, Social Change and Pluralism», IUCN Academy of Environmental Law e-Journal n. 1, 2012, pp. 18-29. Por otra parte, sobre la idea del Derecho como filtro social del desarrollo tecnológico y control social de los riesgos que supone, vid. CALLIES, Ch., Rechtstaat und Umweltstaat, Mohr Siebeck, Tubinga, 2001, p. 65.

${ }^{49}$ En relación con la (posible) emergencia de un constitucionalismo de las responsabilidades, deben mencionarse algunos textos recientes, como la Constitución polaca de 1997, la suiza de 1999 o la Carta francesa del medio ambiente de 2005. La idea de responsabilidad se vincula con la idea de cuidado (care), aportada por el ecofeminismo, de acuerdo con un ethos consciente del impacto de nuestras acciones en el entorno y en los demás, que no solo delimita la libertad, la gran conquista de la modernidad europea, sino que implica un status comprometido de los miembros de la comunidad global. En relación con la noción de cuidado en el ecofeminismo, vid. Held, V., The Ethics of Care: Personal, Political, Global, University of Oxford Press, Oxford, 2006. Finalmente, sobre la vulnerabilidad de la naturaleza y sus implicaciones éticas en relación con lo que aquí se propugna, vid. Jonas, H., El principio de responsabilidad. Ensayo de una ética para la civilización tecnológica cit., pp. 32 ss.
} 
con el objetivo de desprender de los derechos las pulsiones predadoras fundadas en el individualismo posesivo y la religión del progreso, que debería sustituirse por una pragmática adaptación al medio ${ }^{50}$. En este contexto, la idea de responsabilidad se vincula con la calidad de vida para ofrecer un marco de inclusión a los miembros de la comunidad global ${ }^{51}$. De este modo, el paradigma de los derechos que ha presidido el desarrollo de la tradición constitucional desde sus orígenes, debe ser reconducido a una idea de status que unifique las nociones de derechos, responsabilidades y bienestar.

Materialmente, ello debe desplegarse a través del uso del principio de precaución que debe garantizar la responsabilidad en las decisiones políticas de los diferentes actores del sistema y la protección de los más vulnerables ante los riesgos, garantizando, en última instancia, el status de los miembros de la comunidad ${ }^{52}$. Por otra parte, debe asegurarse que el grado de responsa-

${ }^{50}$ La idea de justicia ambiental fue desarrollada, en primer lugar, en Estados Unidos. Dicha noción emergió en los años ochenta, vinculada al movimiento de los derechos civiles y a las demandas en relación con la discriminación racial. El movimiento de la justicia ambiental recibió un impulso muy importante con el manifiesto Principles of Environmental Justice, redactado en el marco de la First National People of Colour Environmental Leadership Summit, que tuvo lugar en octubre de 1991 (vid., por ejemplo, BossELMANN, K., «Justice and the Environment: Building Blocks for a Theory on Ecological Justice», en Bosselmann, K., y Richardson, B. J. (Eds.), Environmental Justice and Market Mechanisms. Key Challenges for Environmental Law and Policy, Kluwer, La Haya-LondresBoston, p. 30). Desde entonces la idea de una distribución injusta de los daños ambientales -y, es cierto, la necesidad de la participación pública en los procesos de toma de decisiones que diseñan las políticas y las regulaciones ambientales- ha sido dominante en el debate sobre la justicia ambiental en Estados Unidos. En este sentido, la definición dada por la Agencia de Protección del Medio Ambiente (EPA) es la siguiente: «Environmental Justice is the fair treatment and meaningful involvement of all people regardless of race, color, national origin, or income with respect to the development, implementation, and enforcement of environmental laws, regulations, and policies. EPA has this goal for all communities and persons across this Nation. It will be achieved when everyone enjoys the same degree of protection from environmental and health hazards and equal access to the decision-making process to have a healthy environment in which to live, learn, and work» (disponible en http://www.epa.gov/environmentaljustice/>, última consulta realizada el $1 \mathrm{de}$ noviembre de 2011). Buenos ejemplos de esta perspectiva centrada en los daños son CoLE, L. W., Foster, S. R., From the Ground Up. Environmental Racism and the Rise of Environmental Justice Movement, New York University Press, Nueva York-Londres, 2001, p. 66; o Cutter, S. L., «Race, class and environmental justice», Progress in Human Geography n. 19-1, 1995, p. 112. Mi punto de vista es más comprensivo (vid. JARIA I MANZANO, J., «Environmental Justice, Social Change and Pluralism» cit., pp. 18-29.).

${ }^{51}$ En relación con la idea de calidad de vida en este contexto, vid. JARIA I MANZANO, J., «Calidad de vida», Diccionario de derechos humanos, Alcalá: Universidad de Alcalá / Agencia Española de Cooperación Internacional para el Desarrollo, $2011<$ http://diccionario.pradpi.org/inicio/index.php/terminos_pub/view/41>, y la bibliografía que allí se cita (última consulta realizada el 9 de octubre de 2012).

${ }_{52}^{5}$ Sobre el principio de precaución, vid., entro muchos otros, Benidickson, J., Environmental Law, Irwin Law, Ottawa, 1997, pp. 18 ss.; CeCchetti, M., Principi costituzionali 
bilidad se corresponda con el grado de poder, de modo que, contrariamente a lo que ahora sucede, los derechos no sirvan para proteger a los más poderosos ante los débiles, sino para garantizar la posición social de los más vulnerables $^{53}$. En este sentido, el centro de gravedad del sistema, donde se define el status de los miembros de la comunidad debe desplazarse de la idea fuerza de los derechos, a la idea fuerza de la responsabilidad ${ }^{54}$.

\section{CONCLUSIÓN}

Los problemas planteados por la reconfiguración global de las relaciones de poder en la fase actual del proceso de acumulación capitalista, cuyo elemento principal es la integración de los mercados pueden y deben tener una respuesta desde el constitucionalismo. En mi opinión, dicha respuesta debe fundarse en tres elementos esenciales, que constituyen, asimismo una cierta actualización de los fundamentos del Derecho constitucional, a saber: la división de poderes - reconstruida alrededor de las ideas de democracias fragmentadas y círculos de consenso-, el control como garantía del status de los miembros de la comunidad -y la consiguiente necesidad de generar estructuras institucionales apropiadas para el nivel global-, y, finalmente, la protección de los miembros de la comunidad -si bien, redefiniendo el papel de los derechos e introduciendo el principio de responsabilidad como elemento regulador-.

per la tutela dell'ambiente, Giuffrè, Milán: 2000, pp. 169 ss.; DE SADELEER, N., «Reflexiones sobre el estatuto jurídico del principio de precaución», Revista de Derecho Ambiental n. 25, 2000, pp. 9-38; ERBGUTH, W., Rechtssystematische Grundfragen des Umweltrechts, Duncker \& Humblot, Berlín, 1987, pp. 92 ss.

${ }^{53}$ Vid. JARIA I MANZANO, J., La cuestión ambiental y la transformación de lo público cit., p. 297.

${ }^{54}$ Vid. Rolston III, H., «Rights and Responsibilities on the Home Planet», Yale Journal of International Law, n. 18, 1993, p. 252. 